\title{
Avaliação da qualidade de vida em pacientes com escoliose idiopática do adolescente após tratamento cirúrgico pelo questionário SF-36
}

\author{
Health-related quality of life in patients with adolescent idiopathic \\ scoliosis after surgical treatment by SF-36
}

Evaluación de la calidad de vida en pacientes con escoliosis idiopática del adolescente después del tratamiento quirúrgico por el cuestionario SF-36

\author{
Luciano Temporal Borges Cabral \\ Edgar Santiago Valesin Filho² \\ Fabrício Hidetoshi Ueno² \\ Adriano Masayuki Yonezaki² \\ Luciano Miller Reis Rodrigues ${ }^{3}$
}

\section{RESUMO}

Objetivo: avaliar os pacientes com escoliose idiopática do adolescente após tratamento cirúrgico, pelo questionário SF-36, comparando-os com questionário realizado no préoperatório. Métodos: entre Fevereiro de 2004 a Março de 2006 foram selecionados 28 pacientes com escoliose idiopática do adolescente, tratados cirurgicamente pela equipe de cirurgia da coluna do Hospital Mário Covas, em Santo André. Todos os pacientes foram submetidos ao tratamento cirúrgico por via posterior e artrodese com instrumentação de terceira geração. Estes pacientes possuíam descompensação do tronco e mensuração do ângulo de Cobb maior que $50^{\circ}$ para indicação cirúrgica. Todos eles foram submetidos ao questionário SF-36 no pré-operatório, este foi repetido em um mês, seis meses,

\section{ABSTRACT}

Objective: to assess health-related quality of life in patients with adolescent idiopathic scoliosis by $S F-36$ after surgical treatment, in comparison with questionnaires done before operation. Methods: between February 2004 and March 2006, 28 patients with adolescent idiopathic scoliosis surgically treated by the spine group of Hospital Mário Covas, in Santo André, were assessed. All the patients were treated surgically by posterior approach and fusion with third generation instrumental. These patients had trunk rotation and Cobb angle more than $50^{\circ}$ to have surgical indication. All patients were submitted to SF-36 before and after one month, six months, one year and two years after surgery. The SPSS (Statistical
\end{abstract}

\section{RESUMEN}

Objetivo: evaluar los pacientes con escoliosis idiopática del adolescente después del tratamiento quirúrgico por medio del cuestionario SF-36, comparándolos con el cuestionario realizado pre operatoriamente. Métodos: entre Febrero de 2004 a Marzo de 2006 fueron seleccionados 28 pacientes con escoliosis idiopática del adolescente tratados quirúrgicamente por el equipo de cirugía de la columna del Hospital Mário Covas, en Santo André. Todos los pacientes fueron sometidos al tratamiento quirúrgico por vía posterior $y$ artrodesis con instrumentación de tercera generación. Estos pacientes poseían descompensación del tronco y medida del ángulo de Cobb mayor que $50^{\circ}$ constituyéndose en indicación quirúrgica. Todos los pacientes fueron sometidos al cuestionario SF-36 preoperatorio y repetidos con un mes,

\footnotetext{
Trabalho realizado no Hospital Mário Covas da Faculdade de Medicina do ABC - FMABC - Santo André (SP), Brasil.

'Médico Colaborador do Grupo de Cirurgia da Coluna da Disciplina de Doenças do Aparelho Locomotor da Faculdade de Medicina do ABC - FMABC - Santo André (SP), Brasil.

${ }_{2}^{2}$ Médico Assistente do Grupo de Cirurgia da Coluna da Disciplina de Doenças do Aparelho Locomotor da Faculdade de Medicina do ABC - FMABC - Santo André (SP), Brasil.

${ }^{3}$ Mestre, Coordenador do Grupo de Cirurgia da Coluna da Disciplina de Doenças do Aparelho Locomotor da Faculdade de Medicina do ABC - FMABC - Santo André (SP), Brasil.
} 
um ano e dois anos após o tratamento cirúrgico. Foi utilizado o programa SPSS (Statistical Package for Social Sciences), versão 13.0 , para a análise estatística dos casos. Resultados: houve diferença estatística significante em quatro domínios do SF-36: capacidade funcional, dor, aspecto social e limitação por aspectos físicos. Nos domínios saúde mental, limitação por aspectos emocionais, vitalidade e estado geral de saúde não houve diferença estatística entre os momentos observados. Conclusão: neste estudo, os pacientes com escoliose idiopática do adolescente, submetidos a tratamento cirúrgico para correção da deformidade, obtiveram melhora da qualidade de vida em aspectos físicos avaliados por meio do questionário SF-36.

DESCRITORES: Qualidade de vida; Escoliose/cirurgia; Adolescente; Resultado de tratamento; Questionários
Package for Social Sciences), version 13.0 for statistical analysis was used. Results: statistical significant differences were noted in four domains of SF-36: physical functioning, bodily pain, role physical and social functioning. In the other four domains, general health, mental health, role emotional and vitality there were no statistical differences in the observed moments. Conclusion: in the present study, the patients with adolescent idiopathic scoliosis, submitted to surgical procedures for deformity correction, had better results in the physical aspects assessed by $S F-36$.

KEYWORDS: Quality of life; Scoliosis/surgery; Adolescent; Treatment outcome; Questionnaires seis meses, un y dos años después del tratamiento quirúrgico. Usamos el programa SPSS (Statistical Package for Social Sciences), versión 13.0, para análisis estadístico de los casos. Resultados: hubo diferencia estadística significativa en cuatro dominios del SF36: capacidad funcional, dolor, aspecto social y limitaciones por aspectos fisicos. En los dominios de salud mental, limitación pos aspectos emocionales, vitalidad y estado general de salud no hubo alguna diferencia estadística entre los momentos observados. Conclusión: en este estudio los pacientes con escoliosis idiopática del adolescente, sometidos al tratamiento quirúrgico para corrección de la deformidad, obtuvieron una mejoría en la calidad de vida en aspectos fisicos evaluados por medio del cuestionario SF-36.

DESCRIPTORES: Calidad de vida; Escoliosis/cirugía; Adolescente; Resultado del tratamiento; Cuestionario

\section{INTRODUÇÃO}

A prevalência global da escoliose é entre 1 a 2\%, e a escoliose idiopática do adolescente (EIA) é o subgrupo mais comum ${ }^{1,2}$.

As consequências clínicas da EIA para os pacientes são predominantemente cosméticas, especialmente em curvas muito severas. Nestes poucos pacientes, a disfunção respiratória e a progressão da curva na vida adulta têm um papel de fundamental importância ${ }^{3}$. O ortopedista possui a órtese e o tratamento cirúrgico para oferecer a estes pacientes ${ }^{4}$. O objetivo do tratamento com órtese é impedir a progressão da curva e evitar fusão da coluna vertebral ${ }^{5}$. O objetivo da cirurgia é impedir a progressão, corrigir a curva e manter o balanço da coluna vertebral com a menor fusão possível ${ }^{6}$.

Weinstein et al. documentaram cuidadosamente a evolução clínica de pacientes com EIA sem tratamento7. Grandes curvas (maiores que $50^{\circ}$ ) sem tratamento progridem substancialmente durante a vida adulta e causam aumento da deformidade; juntamente com a disfunção pulmonar que reduz a expectativa de vida e tem grande impacto na vida diária ${ }^{8}$. Estudos mostraram que há um aumento da frequência de dor lombar, embora não seja suficiente para restringir as atividades ${ }^{8}$.

Durante a última década, o interesse em avaliar como os pacientes percebem o impacto da doença aumentou, isso comumente é referido como qualidade de vida relacionada à saúde. A qualidade de vida relacionada à saúde é definida como uma construção multidimensional que captura o impacto do estado de saúde, incluindo doença e tratamento em três domínios: físico, psicológico e função social ${ }^{9}$. Vários questionários foram desenvolvidos para avaliação da qualidade de vida relacionada à saúde. Neste estudo, realizou-se a avaliação dos pacientes com EIA após tratamento cirúrgico pelo questionário SF-36, comparando-os com um questionário realizado no pré-operatório.

\section{MÉTODOS}

Entre Fevereiro de 2004 a Março de 2006 foram selecionados 28 pacientes com EIA tratados cirurgicamente pela equipe de cirurgia da coluna do Hospital Mário Covas, em Santo André. Todos os pacientes foram submetidos ao tratamento cirúrgico por via posterior e artrodese com instrumentação de terceira geração. Estes pacientes possuíam descompensação do tronco e mensuração do ângulo de Cobb maior que $50^{\circ}$ para indicação cirúrgica. Foram avaliadas 22 meninas e 6 meninos média de idade de 15 anos e 2 meses (13 a 20 anos). Os 28 pacientes apresentavam curvas torácicas e lombares, mas em apenas quatro $(9,53 \%)$ casos a curva lombar era a principal (Tabela 1$)$.

Utilizou-se como critério de inclusão a idade do diagnóstico (dez anos até a maturidade esquelética), presença de escoliose idiopática, indicação cirúrgica de correção por via posterior e a presença de curva lombar e/ou torácica. Já 
TABELA 1 - Características do grupo em estudo quanto ao número, sexo, idade e curva principal

\begin{tabular}{|c|c|c|}
\hline Grupo & & Curva principal \\
\hline Número de pacientes & 28 & \\
\hline \multicolumn{3}{|l|}{ Sexo } \\
\hline Masculino & $6(21,4 \%)$ & \\
\hline Feminino & $22(78,6 \%)$ & \\
\hline Média de idade & $\begin{array}{c}15 \text { anos e } 2 \\
\text { meses (13 a 20) }\end{array}$ & \\
\hline Curvas torácicas & 28 & $24(85,7 \%)$ \\
\hline Curvas lombares & 28 & $4(14,3 \%)$ \\
\hline
\end{tabular}

os critérios de exclusão foram: presença de outra desordem neuromuscular, escoliose não idiopática, cirurgia prévia de coluna, necessidade de associação com via de acesso anterior, estudo radiológico incompleto e de má qualidade.

No presente estudo, a média do ângulo de Cobb pré-operatório para as curvas torácicas foi de $62,38^{\circ}$, enquanto que esta no pós-operatório foi de $38,28^{\circ}$. Já a média dos ângulos de Cobb pré-operatório para as curvas lombares foi de $40,52^{\circ}$, e esta foi de $21,74^{\circ}$ para as mesmas curvas lombares. Esses resultados mostraram uma melhora média de $24,1^{\circ}$ nas deformidades pós-operatórias torácicas, e de $18,78^{\circ}$ nas deformidades lombares. Isso reflete em uma melhora do ângulo de Cobb de $61,36 \%$ para as curvas torácicas e de $53,65 \%$ para as lombares, conforme apresentado na Tabela 2.

TABELA 2 - Medida dos valores pré e pós-operatórios e melhora em graus e em porcentagem do ângulo de Cobb.

\begin{tabular}{lcc}
\hline & $\begin{array}{c}\text { Curva } \\
\text { torácica }\end{array}$ & $\begin{array}{c}\text { Curva } \\
\text { lombar }\end{array}$ \\
\hline Média Cobb pré & $62,38^{\circ}$ & $40,52^{\circ}$ \\
Média Cobb pós & $38,8^{\circ}$ & $21,74^{\circ}$ \\
Melhora do Cobb em graus & $24,1^{\circ}$ & $18,78^{\circ}$ \\
Melhora do Cobb & $61,36 \%$ & $53,66 \%$ \\
\hline
\end{tabular}

Todos os pacientes foram submetidos ao questionário SF-36 pré-operatoriamente e repetidos com um mês, seis meses, um ano e dois anos após o tratamento cirúrgico. O questionário SF-36 para avaliação da qualidade de vida contém 36 itens e mede o componente de saúde mental e de física por oito domínios: capacidade funcional, limitação por aspectos físicos, dor, estado geral de saúde, vitalidade, aspectos sociais, limitação por aspectos emocionais e saúde mental. O SF-36 pode ser autoadministrado pelo computador, telefone ou por um entrevistador treinado e pode ser administrado entre cinco a dez minutos ${ }^{10}$. Neste estudo, a secretária do grupo, formada no curso de fisioterapia, aplicou todos os questionários em dois anos.

Foi utilizado nível de significância de 5\% $(0,050)$ para a aplicação dos testes estatísticos, ou seja, quando o valor da significância calculada (p) for menor do que 5\% $(0,050)$, observa-se uma diferença dita 'estatisticamente significante' (marcada em vermelho); e quando o valor da significância calculada (p) for igual ou maior do que 5\% $(0,050)$, é observada uma diferença dita 'estatisticamente não significante'.

Usou-se o programa SPSS (Statistical Package for Social Sciences), versão 13.0, para a obtenção dos resultados.

\section{RESULTADOS}

Foi aplicado o teste de Friedman, com o intuito de serem verificadas possíveis diferenças entre os cinco momentos de observação, quando comparados concomitantemente, para os oito domínios do SF-36 (Tabelas 3 a 10).

Notou-se diferença estatisticamente significante em quatro domínios do SF-36: capacidade funcional, dor, aspecto social e limitação por aspectos físicos (Tabela 11). Dos quatro domínios que tiveram diferença estatística, a capacidade funcional e a limitação por aspectos físicos obtiveram resultados cada vez melhores com o tempo. Nos domínios saúde mental, limitação por aspectos emocionais, vitalidade e estado geral de saúde não houve diferença estatística entre os momentos observados. Por serem encontradas diferenças estatisticamente significantes para os momentos de observação para quatro domínios, o teste

TABELA 3 - Relação entre a capacidade funcional (CF) com os cinco momentos de observação.

\begin{tabular}{lccccccc}
\hline Variável & $\mathbf{n}$ & Média & Desvio padrão & Mínimo & Máximo & Mediana & Significância (p) \\
\hline CF pré & 28 & 74,52 & 9,34 & 55 & 95 & 75 & \\
CF 1 m & 28 & 60,71 & 19,12 & 15 & 95 & 65 & \\
CF 6 m & 28 & 81,9 & 8,73 & 65 & 95 & 80 & $<0,001$ \\
CF 1 a & 28 & 82,86 & 10,44 & 55 & 95 & 85 & \\
CF 2 a & 28 & 85,24 & 7,5 & 70 & 95 & 85 & \\
\hline
\end{tabular}

TABELA 4 - Relação entre a limitação de aspecto físico (LAF) com os cinco momentos de observação.

\begin{tabular}{lccccccc}
\hline Variável & $\mathbf{n}$ & Média & Desvio padrão & Mínimo & Máximo & Mediana & Significância (p) \\
\hline LAF pré & 28 & 55,95 & 26,11 & 0 & 100 & 50 & \\
LAF 1 m & 28 & 35,71 & 26,89 & 0 & 100 & 50 & \\
LAF 6 m & 28 & 76,19 & 20,12 & 50 & 100 & 75 & $<0,001$ \\
LAF 1 a & 28 & 71,43 & 30,91 & 0 & 100 & 75 & \\
LAF 2 a & 28 & 78,57 & 26,56 & 25 & 100 & 100 & \\
\hline
\end{tabular}


TABELA 5 - Relação entre a dor e os cinco momentos de observação.

\begin{tabular}{lccccccc}
\hline Variável & $\mathbf{n}$ & Média & Desvio padrão & Mínimo & Máximo & Mediana & Significância $(\mathrm{p})$ \\
\hline Dor pré & 28 & 59,05 & 13,75 & 20 & 80 & 60 & \\
Dor 1 m & 28 & 63,81 & 15,32 & 20 & 100 & 60 & \\
Dor 6 m & 28 & 71,9 & 8,14 & 60 & 100 & 70 & $<0,001$ \\
Dor 1 a & 28 & 72,38 & 11,79 & 50 & 100 & 70 & \\
Dor 2 a & 28 & 70,48 & 11,17 & 50 & 100 & 70 & \\
\hline
\end{tabular}

TABELA 6 - Relação entre o estado geral (EG) e os cinco momentos de observação.

\begin{tabular}{lccccccc}
\hline Variável & $\mathbf{n}$ & Média & Desvio padrão & Mínimo & Máximo & Mediana & Significância $(\mathrm{p})$ \\
\hline EG pré & 28 & 64,52 & 16,5 & 35 & 90 & 70 & \\
EG 1 m & 28 & 70,48 & 15,8 & 35 & 95 & 70 & \\
EG 6 m & 28 & 70,95 & 14,2 & 45 & 90 & 75 & 0,08 \\
EG 1 a & 28 & 68,33 & 17,84 & 35 & 90 & 70 & \\
EG 2 a & 28 & 72,14 & 17,43 & 45 & 100 & 75 & \\
\hline
\end{tabular}

TABELA 7 - Relação entre vitalidade (V) e os cinco momentos de observação.

\begin{tabular}{lccccccc}
\hline Variável & $\mathrm{n}$ & Média & Desvio padrão & Mínimo & Máximo & Mediana & Significância $(\mathrm{p})$ \\
\hline V pré & 28 & 69,76 & 15,69 & 30 & 90 & 75 & \\
V 1 m & 28 & 67,86 & 6,04 & 60 & 80 & 65 & \\
V 6 m & 28 & 69,05 & 14,11 & 40 & 85 & 75 & 0,153 \\
V 1 a & 28 & 71,19 & 16,27 & 35 & 90 & 75 & \\
V 2 a & 28 & 72,38 & 14,88 & 45 & 90 & 80 & \\
\hline
\end{tabular}

TABELA 8 - Relação entre aspecto social (AS) e os cinco momentos de observação.

\begin{tabular}{lccccccc}
\hline Variável & $\mathbf{n}$ & Média & Desvio padrão & Mínimo & Máximo & Mediana & Significância $(\mathbf{p})$ \\
\hline AS pré & 28 & 76,79 & 12,68 & 50 & 100 & 75 & \\
AS 1 a & 28 & 75,6 & 13,39 & 50 & 100 & 75 & \\
AS 6 a & 28 & 89,88 & 12,88 & 50 & 100 & 87,5 & $<0,001$ \\
AS 1 a & 28 & 95,24 & 8,36 & 75 & 100 & 100 & \\
AS 2 a & 28 & 88,1 & 19,56 & 37,5 & 100 & 100 & \\
\hline
\end{tabular}

TABELA 9 - Relação entre limitação no aspecto emocional (LAE) e os cinco momentos de observação.

\begin{tabular}{lccccccc}
\hline Variável & $\mathbf{n}$ & Média & Desvio padrão & Mínimo & Máximo & Mediana & Significância $(\mathrm{p})$ \\
\hline LAE pré & 28 & 85,71 & 19,92 & 33,33 & 100 & 100 & \\
LAE 1 m & 28 & 80,95 & 19,92 & 33,33 & 100 & 66,67 & \\
LAE 6 m & 28 & 87,3 & 22,3 & 33,33 & 100 & 100 & $<0,541$ \\
LAE 1 a & 28 & 90,48 & 18,69 & 33,33 & 100 & 100 & \\
LAE 2 a & 28 & 84,13 & 24,99 & 33,33 & 100 & 100 & \\
\hline
\end{tabular}


TABELA 10 - Relação entre saúde mental (SM) e os cinco momentos de observação.

\begin{tabular}{lccccccc}
\hline Variável & $\mathbf{n}$ & Média & Desvio padrão & Mínimo & Máximo & Mediana & Significância $(\mathbf{p})$ \\
\hline SM pré & 28 & 72,19 & 17,09 & 28 & 92 & 76 & \\
SM 1 m & 28 & 73,9 & 11,29 & 48 & 92 & 76 & \\
SM 6 m & 28 & 76,19 & 16,85 & 28 & 92 & 84 & 0,431 \\
SM 1 a & 28 & 70,1 & 16,08 & 28 & 88 & 76 & \\
SM 2 a & 28 & 70,86 & 17,94 & 40 & 92 & 76 & \\
\hline
\end{tabular}

TABELA 11 - Demonstração estatística para identificar quais momentos de observação diferenciamse entre si, quando comparados par a par, dos domínios com diferença estatisticamente significante.

\begin{tabular}{|c|c|c|c|c|}
\hline \multirow[b]{2}{*}{ Par de momentos } & \multicolumn{4}{|c|}{ Domínio do SF-36 } \\
\hline & Aspecto social & Capacidade funcional & Dor & $\begin{array}{l}\text { Limitação por } \\
\text { aspectos físicos }\end{array}$ \\
\hline PRE $\times 1 M$ & 0,72 & 0,01 & 0,254 & 0,041 \\
\hline PRE $\times 6 M$ & 0,006 & 0,006 & 0,002 & 0,029 \\
\hline PRE $\times 1 A$ & 0,001 & 0,015 & 0,001 & 0,129 \\
\hline PRE $\times 2 A$ & 0,058 & 0,001 & 0,011 & 0,027 \\
\hline $1 M \times 6 M$ & 0,004 & $<0,001$ & 0,004 & $<0,001$ \\
\hline $1 \mathrm{M} \times 1 \mathrm{~A}$ & $<0,001$ & $<0,001$ & 0,019 & 0,001 \\
\hline $1 M \times 2 A$ & 0,007 & $<0,001$ & 0,037 & $<0,001$ \\
\hline $6 \mathrm{M} \times 1 \mathrm{~A}$ & 0,123 & 0,597 & 0,855 & 0,543 \\
\hline $6 \mathrm{M} \times 2 \mathrm{~A}$ & 0,684 & 0,025 & 0,55 & 0,467 \\
\hline $1 \mathrm{~A} \times 2 \mathrm{~A}$ & 0,072 & 0,145 & 0,417 & 0,141 \\
\hline
\end{tabular}

PRE: pré-operatório; M: mês; A: ano.

dos postos sinalizados de Wilcoxon será aplicado para identificar quais momentos de observação diferenciam-se entre si, quando comparados par a par.

Analisando os momentos de observação entre si, concluiu-se que existe diferença estatística entre o préoperatório e o seguimento de dois anos nos domínios dor, capacidade funcional e limitação por aspectos físicos.

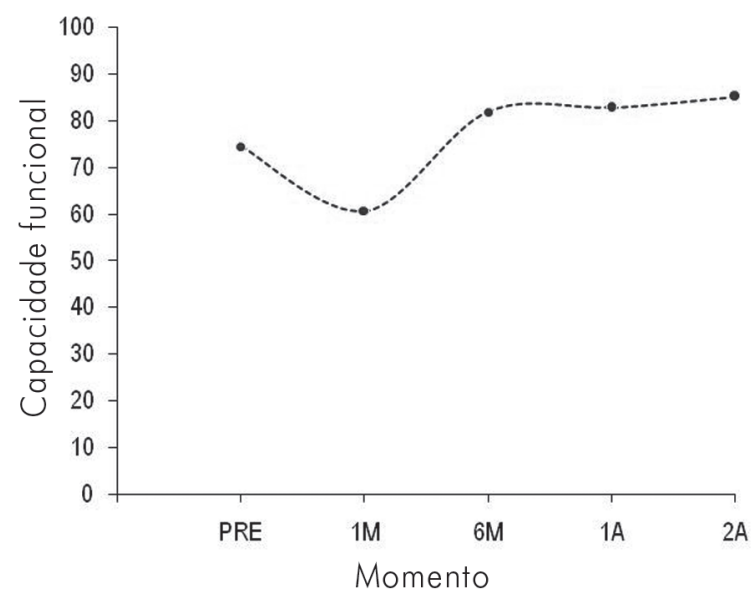

Gráfico 1

Relação entre capacidade funcional e os cinco momentos da observação.
Em relação ao pós-operatório de um mês e o seguimento de dois anos, mostrou-se que existe diferença estatística nos quatro domínios. Assim como no pós-operatório de um mês em relação ao seguimento de seis meses, e entre o pós-operatório de um mês e um ano. A representação gráfica da relação entre cada domínio e seus respectivos momentos de observação é observada nos Gráficos 1 a 8.

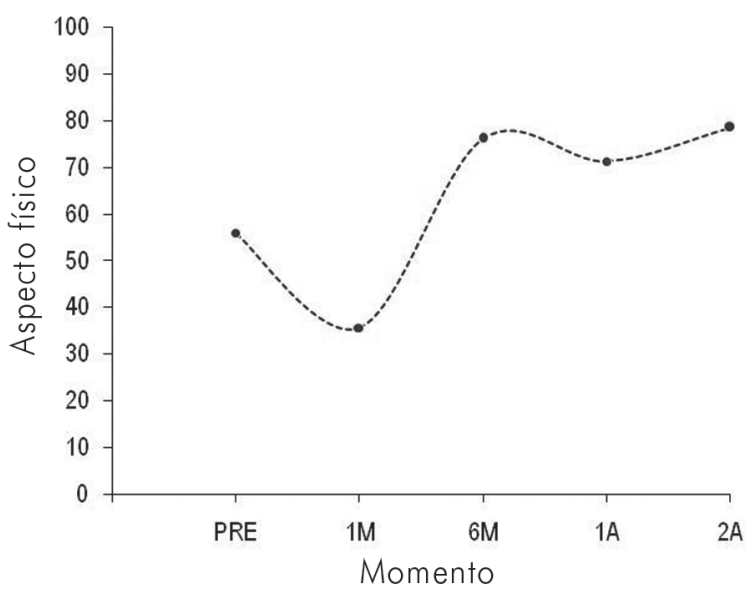

Gráfico 2

Relação entre aspecto físico e os cinco momentos de observação. 


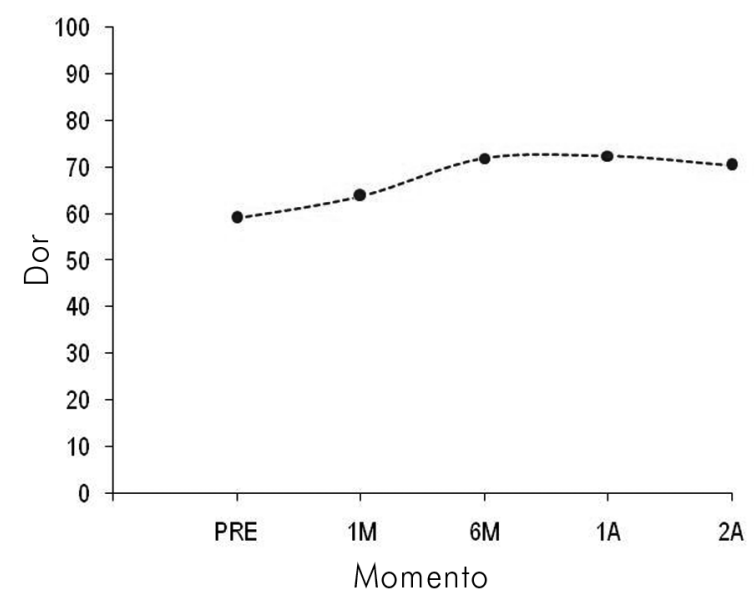

Gráfico 3

Relação entre dor e os cinco momentos de observação.

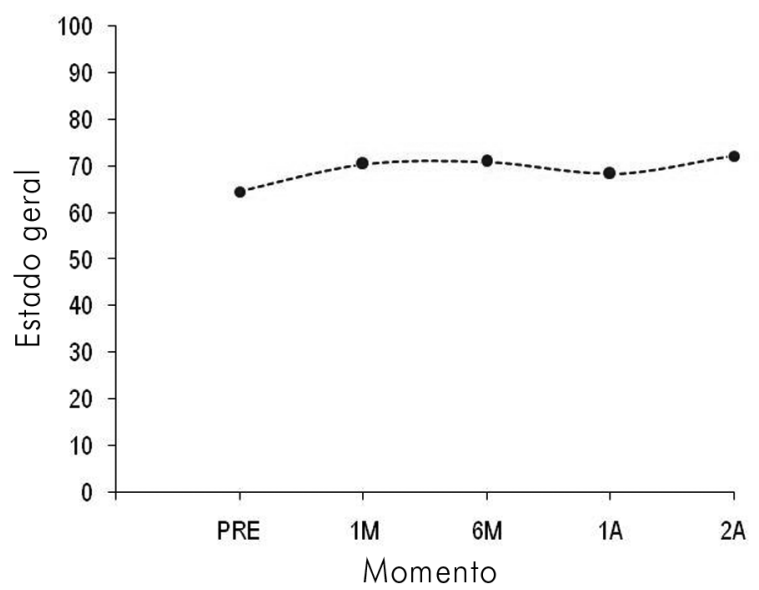

Gráfico 4

Relação entre estado geral e os cinco momentos de observação.

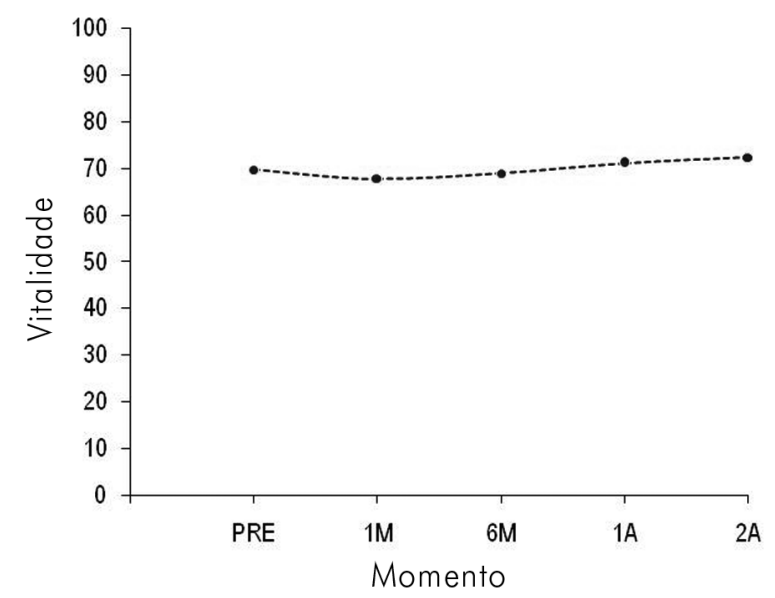

Gráfico 5

Relação entre vitalidade e o cinco momentos de observação.

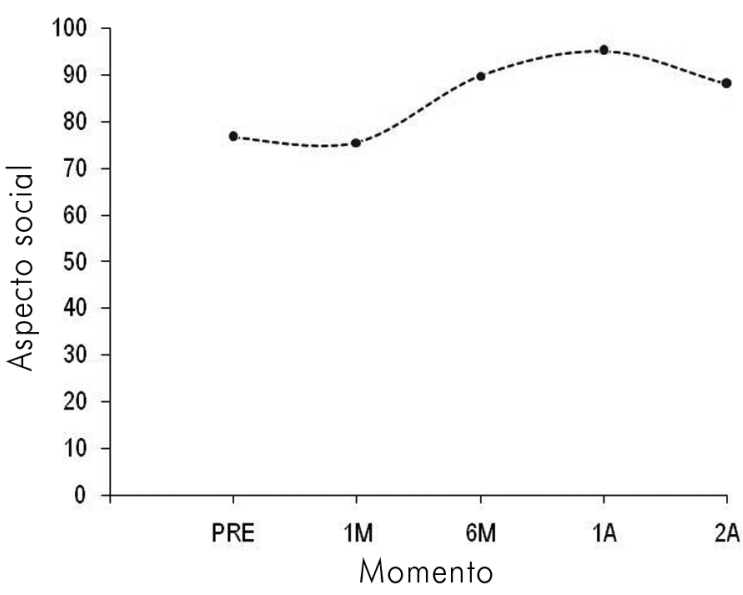

Gráfico 6

Relação entre aspecto social e os cinco momentos de observação.

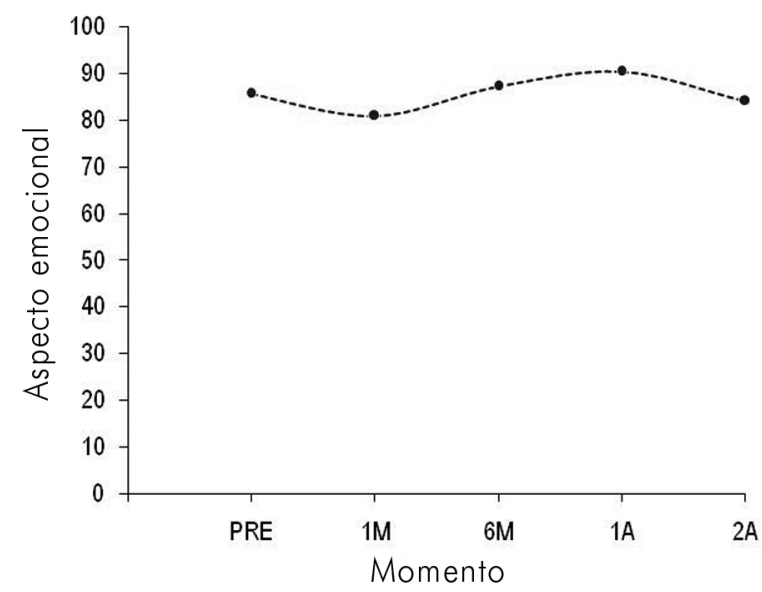

Gráfico 7

Relação entre aspecto emocional e os cinco momentos de observação.

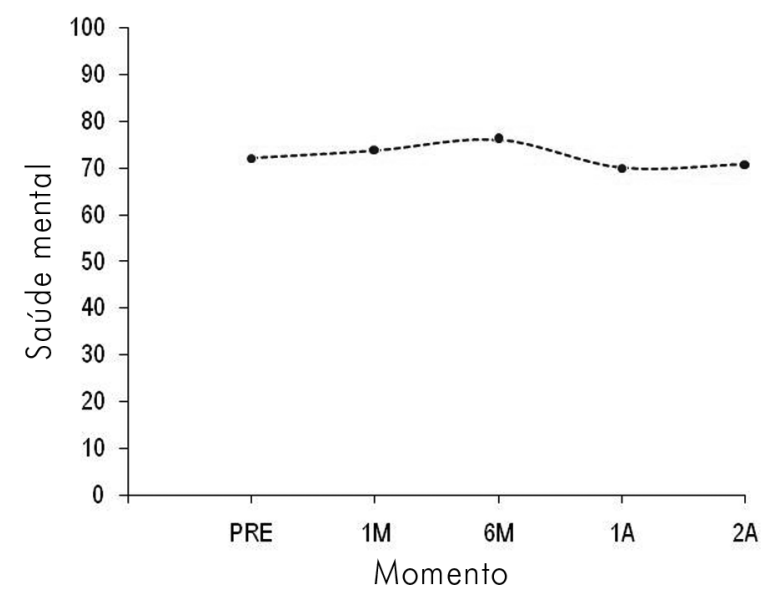

Gráfico 8

Relação entre saúde mental e os cinco momentos de observação. 


\section{DISCUSSÃO}

No presente artigo, utilizou-se o questionário SF-36 (resultados médicos de confiança, Boston, Ma) na avaliação da qualidade de vida dos pacientes portadores de EIA, devido a sua praticidade em ser aplicado e, segundo Ware, sua utilidade foi ilustrada em artigos para mais de 130 doenças e condições ${ }^{10}$. Já se documentou em diversos estudos que problemas relacionados à coluna vertebral podem afetar significativamente a qualidade de vida relacionada à saúde e a avaliação pode ser feita pelo SF-36 ${ }^{11}$. Por meio de análise estatística, observou-se que houve melhora significativa no aspecto físico dos pacientes pós-cirúrgicos com seguimento de dois anos em relação à antes da operação. Essa melhora também foi observada no trabalho de Ware e Sherbourne, o qual mostrava diferença estatística entre pacientes operados e um Grupo Controle ${ }^{12}$.

Na literatura, dor na coluna é uma importante questão relacionada com escoliose idiopática $(8,73 \%$ mais que qualquer outro critério, não incluso o grau Cobb $)^{13}$. $\mathrm{Na}$ adolescência, aproximadamente $30 \%$ dos pacientes apresentam dor na coluna ${ }^{14}$. Em nosso estudo obtivemos melhora estatística da dor em pacientes operados com seguimento de dois anos, em relação aos pré-cirúrgicos. Este resultado foi semelhante ao estudo de Haher et al., o qual demonstrou que a relação mais forte nos pacientes operados relativos aos mesmos pacientes antes da cirurgia foi a dor seguida pela melhora da imagem ${ }^{15}$. No nosso estudo também houve melhora estatística do domínio aspecto social, podendo relacioná-lo com melhora da imagem, assim como no trabalho de Haher et al. ${ }^{15}$.

Diferentemente de outros trabalhos encontrados na literatura, este estudo mostrou melhora significativa em aspectos físicos, mas não mostrou melhora em aspectos de saúde mental. Kibsgård et al. avaliaram pelo SF-36 em pacientes noruegueses com EIA, submetidos à cirurgia com seguimento de dois a três anos, resultados inferiores em relação aos aspectos físicos e resultados superiores em relação aos aspectos de saúde mental comparados ao nosso estudo ${ }^{16}$.

Foram encontradas diferenças estatisticamente significantes para os momentos de observação nos domínios: dor, capacidade funcional, limitação de aspecto físico e aspecto social. Aplicando-se o teste dos postos sinalizados de Wilcoxon, para identificarmos quais momentos de observação diferenciam-se entre si, quando comparados par a par alguns resultados interessantes foram encontrados.

Quando comparados esses quatro domínios no préoperatório com o pós-operatório de um mês, apenas os domínios capacidade funcional e limitação por aspecto físico tiveram melhora estatisticamente significativa, já a dor e o aspecto social não obtiveram melhora. Entretanto, quando comparados os quatro domínios entre o pós-operatório de um mês e seis meses, um mês e um ano e um mês e dois anos, todos resultaram numa melhora significativa. O que nos leva a entender que entre o pré-operatório e um mês após a cirurgia o paciente ainda apresenta algum desconforto como dor, ferida operatória apresentando deiscência. Já após um mês, o paciente apresenta melhora dos desconfortos e não apresenta dificuldades na realização de atividades diárias e com sua nova aparência física. Outro resultado obtido foi que pela comparação entre seis meses e um ano e seis meses com dois anos, não houve melhora significativa nos quatro domínios, assim como nos momentos um ano com dois anos. Isso mostra que houve uma adaptação positiva dos pacientes em relação aos quatro domínios, ou seja, eles se acostumaram com a nova aparência e melhora nas atividades diárias.

\section{CONCLUSÃO}

Nos pacientes com EIA submetidos a tratamento cirúrgico para correção da deformidade ocorreu melhora da qualidade de vida, analisada por meio do questionário SF-36.

\section{REFERÊNCIAS}

1. Lonstein JE, Bjorklund S, Wanninger $\mathrm{MH}$, Nelson RP. Voluntary school screening for scoliosis in Minnesota. $\mathrm{J}$ Bone Joint Surg Am. 1982;64(4):481-8.

2. Andersen MO, Thomsen K, Kyvik KO. Adolescent idiopathic scoliosis in twins: a population-based survey. Spine (Phila Pa 1976). 2007;32(8):927-30.

3. Weinstein SL, Ponseti IV. Curve progression in idiopathic scoliosis. J Bone Joint Surg Am. 1983;65(4): 447-55.

4. Andersen MO, Christensen SB, Thomsen K. Outcome at 10 years after treatment for adolescent idiopathic scoliosis. Spine (Phila Pa 1976). 2006;31(3):350-4.
5. Carr WA, Moe JH, Winter RB, Lonstein JE. Treatment of idiopathic scoliosis in the Milwaukee brace. J Bone Joint Surg Am. 1980;62(4):599-612.

6. Nachemson AL, Peterson LE. Effectiveness of treatment with a brace in girls who have adolescent idiopathic scoliosis. A prospective, controlled study based on data from the Brace Study of the Scoliosis Research Society. J Bone Joint Surg Am. 1995;77(6):815-22.

7. Weinstein SL, Dolan LA, Spratt KF, Peterson KK, Spoonamore MJ, Ponseti IV. Health and function of patients with untreated idiopathic scoliosis: a 50 -year natural history study. JAMA. 2003;289(5):559-67.
8. Howard A, Donaldson S, Hedden D, Stephens D, Alman B, Wright J. Improvement in quality of life following surgery for adolescent idiopathic scoliosis. Spine (Phila Pa 1976). 2007;32(24):2715-8.

9. Leidy NK, Revicki DA, Genesté B. Recommendations for evaluating the validity of quality of life claims for labeling and promotion. Value Health. 1999;2(2):113-27.

10. Ware JE Jr. SF-36 health survey update. Spine (Phila Pa 1976). 2000;25(24):3130-9. Review. 
11. Patrick DL, Deyo RA, Atlas SJ, Singer DE, Chapin A, Keller RB. Assessing health-related quality of life in patients with sciatica. Spine (Phila Pa 1976). 1995;20(17):1899-908; discussion 1909.

12. Ware JE Jr, Sherbourne CD. The MOS 36-item short-form health survey (SF36) I. Conceptual framework and item selection. Med Care. 1992;30(6):473-83.

13. Negrini S, Grivas TB, Kotwicki T, Maruyama T, Rigo M, Weiss HR; Members of the Scientific Society On Scoliosis Orthopaedic and Rehabilitation Treatment (SOSORT). Why do we treat adolescent idiopathic scoliosis? What we want to obtain and to avoid for our patients. SOSORT 2005 Consensus paper. Scoliosis. 2006;1:4.
14.Ramirez N, Johnston CE, Browne $\mathrm{RH}$. The prevalence of back pain in children who have idiopathic scoliosis. J Bone Joint Surg Am. 1997;79(3):364-8.

15. Haher TR, Gorup JM, Shin TM, Homel P, Merola AA, Grogan DP, et al. Results of the Scoliosis Research Society instrument for evaluation of surgical outcome in adolescent idiopathic scoliosis. A multicenter study of 244 patients. Spine (Phila Pa 1976). 1999;24(14):1435-40.

16.Kibsgård T, Brox JI, Reikerås O. Physical and mental health in young adults operated on for idiopathic scoliosis. J Orthop Sci. 2004;9(4): 360-3.

\section{Correspondência}

Luciano Miller Reis Rodrigues

Rua Tucumã, 199, apto. 141 - Jardim

Europa

CEP: 01455-010 - São Paulo (SP), Brasil.

E-mail: luciano.miller@uol.com.br 